GRANDPARENT CAREGIVERS' RESILIENCE: STRESS, SOCIAL SUPPORT, AND COPING PREDICT LIFE SATISFACTION

N. Mendoza, C. Fruhauf, D. MacPhee, Colorado State

University

It is well documented that grandparents raising grandchildren experience high levels of stress, which combined with social isolation and inadequate social support may contribute to grandparent caregivers' low life satisfaction. Social support and coping skills may moderate stress outcomes and thus promote resilience in grandparent caregivers. Thus, this study's purpose was to test a model of resilience in which stress, coping, and social support are predictors of life satisfaction. Grandparents $(\mathrm{N}=74)$ who were raising grandchildren for a minimum of one year completed measures of daily hassles, social support, coping skills, and life satisfaction. Age, marital status, and income were covariates. Descriptive analyses showed that grandparent caregivers are a vulnerable population: high in stress (e.g., feeling overloaded), low in social support, and relatively low in coping and life satisfaction. Structural equation modeling was used to test direct versus moderated effects on life satisfaction. Findings supported direct effects of social support and coping on life satisfaction, but not moderation, and stress no longer predicted life satisfaction with the protective factors in the model, $\mathrm{R} 2=.55$. Additionally, age was a significant covariate, but not the other covariates. These findings provide evidence that social support and coping skills may mitigate the effects of stress on grandparent caregivers, which has implications for interventions to assist grandparents who assume this challenging role.

\section{HOW DO OLDER WORKERS EXPERIENCE INFORMAL CARE PROVISION? THE ROLE OF ACCESS TO PERSONNEL POLICIES}

\section{O. Grunwald, K. Henkens, M. Damman, Netherlands}

Interdisciplinary Demographic Institute (NIDI)

With the increasing retirement age, more older adults have to combine employment with informal caregiving responsibilities. Little is known, however, about how older workers experience caregiving activities next to their paid jobs. This study aims to fill this gap by examining the relationship between work context and both positive and negative caregiving experiences. Using data from the NIDI Pension Panel Survey $(\mathrm{N}=6,793)$, we study caregiving experiences - i.e., the extent to which diverse caregiving activities are satisfactory, burdensome, and stressful - of 1,521 Dutch older workers (age 60-65) who provide care at least once a week. The key predictors concern the work situation, and include, amongst others, access to different personnel policies. Descriptive results show that 70 percent of these older workers experience caregiving as satisfactory, 26 percent as burdensome, and 16 percent as stressful. Multivariate analyses reveal that access to personnel policies plays an explanatory role in the caregiving experience above and beyond work characteristics (e.g., job level, work hours), caregiving situation (e.g., type of care), and individual resources (e.g., health, wealth). Older workers with access to working time adaptation policies experience caregiving as relatively more satisfactory, less burdensome, and less stressful. Older workers with access to regular medical check-ups experience caregiving as less burdensome and less stressful than older workers without access to medical check-ups. Overall, these findings suggest that access to personnel policies, particularly policies that allow for adapting working hours prior to retirement, can facilitate combining work and caregiving obligations in light of longer working lives.

\section{MALES CARING FOR MALES: ARE THEY DIFFERENT FROM OTHER CAREGIVING GENDER DYADS?}

\section{T. Blank ${ }^{1}$, L. Descartes ${ }^{2}, 1$. University of Connecticut, 2.}

Brescia University College

Research on gender differences in informal caregiving primarily has focused on contrasting the experiences of female versus male caregivers. This study focuses on how the gender composition of the caregiver-care-receiver dyad affects the caregiving experience, using male-male dyads as the comparison point. We used National Caregivers Survey data to examine male caregiver-male care-receiver dyads and to contrast them to other gender combinations. For the latter analyses we used one-way ANOVAs of "dyad type" with specific comparisons of male-male dyads $(\mathrm{N}=214)$ to the other three types (total N=1,607; male-male 13.3\%). Average age of male dyad caregivers was 51.4 , about half were primary caregivers, mean weekly hours were 18 , predominant carereceiver need was from disability, and most care-receivers were family members. There were large, significant differences in caregiving hours, perceived burden, emotional strain, primary caregiving, and number of IADLs aided. In some analyses, male-male and female-female dyads were similar to each other in manifesting significant differences from mixed gender dyads. Importantly, differences between same- and different-gender dyads disappeared when only non-spousal/ partner family care-receivers were included. Thus, differences between male-male dyads and others are greatly modified by excluding spousal/partner caregiving from analyses, indicating that spousal/partner caregiving is distinctly different from other caregiving. Research that omits the caregivercare-receiver gender dyad type may incorrectly conclude that males caregive differently from females (e.g., less emotionally involved, provide different aid amounts for IADLs or ADLs), when in reality differences are primarily because of the much lesser likelihood of spousal/partner caregiving in same-gender dyads.

\section{OBSERVED PARENTING ASPECTS OF CHILD COMPLIANCE IN CUSTODIAL GRANDFAMILIES}

L. Portner ${ }^{1}$, P. Kaminski², B. Hayslip², G. Smith ${ }^{3}, 1$.

Behavioral Health Consultants, Austin, TX, 2. University of North Texas, 3. Kent State University

For a myriad of reasons, custodial grandchildren are generally at greater risk of behavioral and emotional difficulties, such as hyperactivity-inattention (HI) (Smith \& Palmieri, 2007). Custodial grandmothers $(N=170)$ completed selfreport measures assessing child $\mathrm{HI}$, expressive social support, and custodial grandmother-grandchild compliance variables. Grandmothers and their grandchildren aged 4 through 12 also completed an observational task that captured their interactions. A multivariate analysis of covariance tested differences between high and low hyperactivity-inattention on observed parenting variables while controlling for child age. As there were significant differences between child age and 
observed parenting variables, a subsequent hierarchical regression model revealed that when controlling for age, child hyperactivity-inattention did not moderate the relationship between commands given by a custodial grandmother and child compliance, but instead revealed that direct commands from the grandmother predicted child compliance. A second hierarchical regression model suggested that encouragement and praise (versus criticism and discouragement) from a grandmother functioned differently across types of commands given, when controlling for child age. It appeared that when grandmothers gave indirect commands more frequently, encouragement and praise instead of criticism was associated with greater compliance. In dyads with frequent direct commands given, compliance was high. However children in dyads who scored high in direct commands accompanied by criticism and discouragement were most likely to comply. This study adds to the knowledge base regarding the nature of grandmother caregiver-grandchild interaction by providing insight into the child-rearing challenges and strengths faced by grandmothers in this unique population.

\section{PATHWAYS OF THE CAREGIVER BURDEN-HEALTH RELATIONSHIP IN FAMILY CAREGIVERS OF VETERANS}

M. Scicolone ${ }^{1}$, P. Parmelee ${ }^{2}$, 1. University of Alabama, 2.

The University of Alabama

The family caregiver population has grown over the years, and while research literature also continues to grow, much less is known specifically about caregivers of veterans. Thus, the current study examines constructs of caregiver burden, self-efficacy, and perceived social support in relation to physical and mental health in caregivers of veterans. For informal family caregivers, perceived capability caring for a loved one can strongly affect the caregiving experience; those with greater self-efficacy likely have lower levels of burden, depression, burnout, heart disease, and increased overall well-being. Additionally, perceived social support is an important component impacting the burden-health relationship, as it is strongly associated with physical and emotional well-being. Using a moderated mediation model, pathways of the burden-health relationship will be examined, with self-efficacy as a mediator of this relationship, and perceived social support as a moderator on the burden-self-efficacy relationship and burden-health relationship. Hypotheses were tested in a sample of 237 family caregivers of elderly veterans receiving outpatient care at the Atlanta Veterans Affairs Medical Center, with the study supported by the U.S. Department of Veterans Affairs Rehabilitation R\&D Service. Preliminary OLS regression analyses revealed two significant overall models $(\mathrm{p}<.001)$ regressing both physical and mental health on predictor variables. Burden significantly predicted both physical and mental health; self-efficacy and perceived social support also significantly predicted mental health. Further analyses examined moderating effects using Process Macro (Hayes, 2017), as well as examination of unique differences of self-efficacy dimensions.

\section{PLANS HELD BY OLDER CAREGIVERS FOR THE FUTURE CARE OF RELATIVES WITH SERIOUS MENTAL ILLNESS}

T. Labrum, Division of Social Work, University of Wyoming
Relatives often provide considerable levels of caregiving to persons with serious mental illness (SMI), with at least onethird of said caregivers being 60 years of age and older. Many older caregivers do not have plans in place for the future care of their relatives with SMI. While the absence of such plans is known to be associated with increased psychological distress for caregivers, little research has examined factors related to the absence of such plans. Using a U.S. community recruited sample of 717 older $(60+)$ caregivers for persons with SMI, the present analysis examines factors associated with the absence of plans for future care. In the present sample, $63 \%$ ( $n=451$ ) of older caregivers reported not having a plan for the future care of their relative with SMI. Results of multivariate logistic regression indicate that the absence of plans is more likely when caregivers report being the sole caregiver and have more difficulty talking with others about the care recipients' mental illness, have feared that the care recipient would intentionally damage their property, and experience more emotional distress as a result of caregiving. Inversely, the absence of plans is less likely when caregivers serve as legal guardians for care recipients. Caregiving provided by older persons towards relatives with SMI receives scant attention from researchers and practitioners alike. It is imperative that practitioners begin assessing whether older caregivers of persons with SMI have plans in place for future care and, when necessary, assist caregivers in creating such plans.

\section{PROFESSIONALS WORKING WITH KINSHIP} FAMILIES: INFORMATIONAL GAPS AND NEEDS

\section{A. Smith, Western Michigan University}

Numbers of grandparents raising children continue to rise. Over 2.7 million American children are in grandparents' care. A 7\% increase during this decade is driven in part by the current opioid drug crisis. Varied multi-generational issues common in kinship families leads to involvement by professionals, representing diverse fields including education, social work, and health care. Despite increased access due to rising numbers, there has been little focus on enhancing professionals' work with kinship families. A pilot study ( $\mathrm{N}=42$ ) explored professionals' experience and preparation for working with kinship families. Over $90 \%$ were employed as teachers, counselors or child welfare workers. All had previously worked with kinship family members. The study assessed participants' previous experience working with kin families, types of information/services provided, readiness for kinship work, access to training, and desire for additional information. Over $85 \%$ of participants reported receiving no professional training or information. $96 \%$ of participants rated themselves as "not very well" or "not at all" prepared to work effectively with kinship family members. Services participants provided to kinship families reflected a diverse focus, including information on child development, parenting skills, access to resources and family relationships. $100 \%$ of participants desired additional information and training in areas including challenges for custodial grandparents, children living in kinship homes, legal and mental health resources, and strategies for linking kinship family members with other kinship families. Results can be used to highlight needs and motivation of professionals for training and information that will enhance their work with kinship families. 